\title{
Molecular Imaging of Rat Cerebellum by Coupling Sub-Monolayer Nanocluster Matrix Implantation, Laser Microprobe Desorption, and Ion-Mobility oTOF-MS.
}

J.Albert Schultz, * Ernest K. Lewis,* Thomas F. Egan,* Valerie Vaughn,* Kelley Waters, ${ }^{*}$ Michael McCully*, Jerry F. Moore, ${ }^{* *}$ Alice Delvolve, ${ }^{* * * J e r e m y ~ P o s t * * *, ~ a n d ~ A m i n a ~ S . ~ W o o d s, ~ * * * ~}$

* Ionwerks, Inc., Houston TX, 77002

** MassThink, Naperville, IL 60563-3281

*** National Institutes on Drug Abuse (NIDA, IRP, NIH) Baltimore, MD, 21224

Molecular imaging of biological tissues by microprobe mass spectrometry is becoming a useful tool-especially when laser microprobe desorption is coupled with organic acid matrix assisted laser desorption/ionization (MALDI-imaging mass spectrometry). MALDI imaging enables identification of intact large molecules from specific locations on a surface [1]. However, the existing spatial resolution (around 10 microns or more) of MALDI imaging by laser microprobe have little to do with the wavelength limitations of the laser focal spot and instead with problems of matrix application to the surface. The in-homogeneity of matrix coverage coupled with uncontrolled solvent extraction from unknown areas of the tissue results in dispersion of molecular information during matrix addition.

The ultimate goal of the laser microprobe imaging of biological tissues is its coupling with, and augmentation of, undeniably useful yet subjective traditional histology. Therefore we have first defined the major problems in existing approaches to molecular imaging and have proceeded to develop analytical methods and instrumentation to solve them. Two of the main problems are: (1) MALDI mass spectra are complicated and have many unresolved isobaric ions (same $\mathrm{m} / \mathrm{z}$ but different compounds, e.g. lipids and peptides). (2) Dried droplet or aerosolized droplet deposition of matrices results in selective solvent extraction of analyte and poor surface coverage uniformity. These two problems have been addressed by a recent combination of ion mobility separation coupled with AuNP implantation into a quite promising experimental approach[2] [3]. The third problem with MALDI imaging is: Mostly neutral biological molecules are desorbed by MALDI from a surface (up to $10^{5}$ neutrals/ion). Consequently we have added a second laser for postionization of these predominant neutrals. Post-ionization not only produces enhanced sensitivity at each pixel but creates analyte ions from many ions which are not detected by MALDI at all.

Nanoparticulate (NP) matrices are implanted into the surface and sub-surface region of tissue sections allowing molecular analysis by laser desorption ionization (MILDI). When MILDI is combined with Ion-Mobility o-TOF MS, a new tool emerges in which the spatial resolution of the microprobe is in principle no longer dependent on matrix uniformity for tissue imaging or intracellular spot profiling of ions and neutrals. Submonolayer AuNP soft-landing and AuNP implantation of sagital rat brain tissue sections was followed by profiling and imaging of lipids in these tissue using MILDI-IonMobilty-oTOFMS. A broad beam ion source effectively implants NP ions into tissue with sub-monolayer uniformity and excellent deposition control. The MILDI-IMoTOF-MS is particularly potent for separating analytically useful ions from elemental or cluster interferences generated from the NP matrix. 
Tissue images were acquired using our standard MALDI-IM-oTOF-MS system [3] comprising an xy stage, a desorption laser, post-ionization laser, a $15 \mathrm{~cm}$ ion mobilty-drift cell operating at $2.3 \mathrm{mb} \mathrm{He}$ atmosphere with a peak resolution of 30, and a high-speed oTOF-MS operating up to $10 \mathrm{kHz}$ which acquires a series of mass spectra as a function of ion mobilty elution time with a mass resolution of 3000. This combination of short drift cell and high-speed TOF allow for a time efficient imaging of a tissue surface in several hours. An example of a single lipid distributed within a rat cerebellum is shown as a purple overlay on top of a photograph of a sagital section of rat brain. The white and grey matter of the brain are evident in both images.
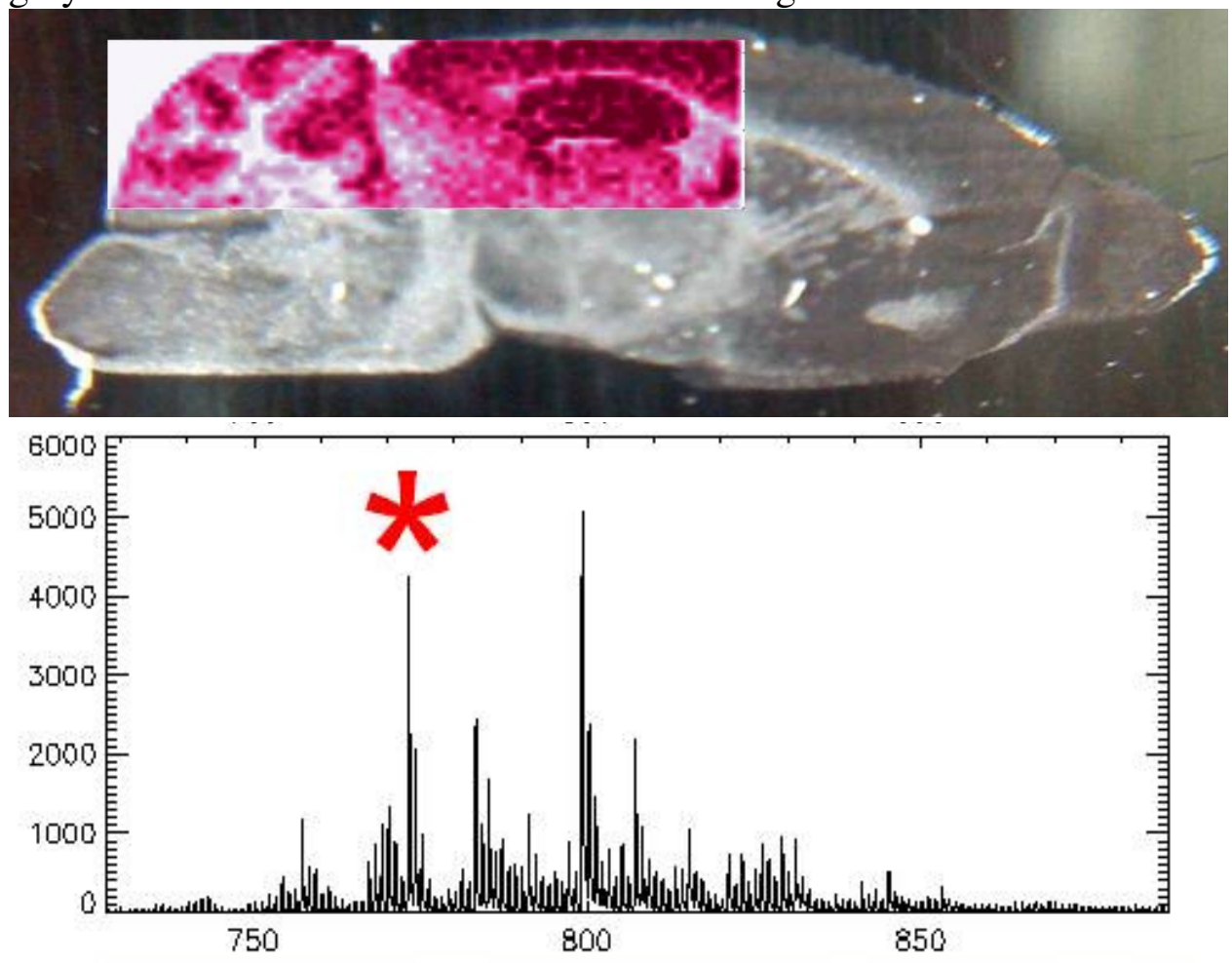

Imaging conditions are 20x80 pixels at 100x100 microns/pixel with 5sec of data acquisition/pixel for a total acquisition time of $2 \mathrm{hrs}$. A mass range of 700 to 900 daltons was selected by presetting a mobility drift cell offset of $0.54 \mathrm{~ms}$ to optimize signal in the range of tissue lipids. MALDI laser irradiation $(349 \mathrm{~nm})$ at $200 \mathrm{~Hz}$ with a spot size of approximately 50 microns produced $\sim 0.06 \mathrm{~mW}$ fluence on the sample. (Asterisk marks $\mathrm{m} / \mathrm{z}$ location of the lipid used to produce the above image.)

\section{References:}

[1] Caprioli, et al., Nature Methods, Vol.4, No10, October 2007

[2] Tempez, Woods, et al., Journal of Proteome Research 2005, 4, 540-545

[3] Jackson, Woods, et al., Journal of Mass Spectrometry, 2007, 42, 1093-1098

[4] Ionwerks gratefully acknowledges support of this research in part through an SBIR contract from the NIH-NIDA through contract HHSN271200900009C (ADB Contract No. N44DA-9-7764). 